\title{
Radio emissions from pulsar companions: a refutable explanation for galactic transients and fast radio bursts
}

\author{
F. Mottez ${ }^{1}$ and P. Zarka ${ }^{2}$ \\ 1 LUTH, Observatoire de Paris, CNRS, Université Paris Diderot, 5 place Jules Janssen, 92190 Meudon, France \\ e-mail: fabrice.mottez@obspm.fr \\ 2 LESIA, Observatoire de Paris, CNRS, UPMC, Université Paris Diderot, 5 place Jules Janssen, 92190 Meudon, France \\ e-mail: philippe.zarka@obspm.fr
}

Received 30 April 2014 / Accepted 28 July 2014

\begin{abstract}
Context. The six known highly dispersed fast radio bursts are attributed to extragalactic radio sources that are of unknown origin but extremely energetic. We propose here a new explanation that does not require an extreme release of energy and involves a body (planet, asteroid, white dwarf) orbiting an extragalactic pulsar.

Aims. We investigate a theory of radio waves associated with such pulsar-orbiting bodies. We focus our analysis on the waves emitted from the magnetic wake of the body in the pulsar wind. After deriving their properties, we compare them with the observations of various transient radio signals to determine whether they could originate from pulsar-orbiting bodies.

Methods. The analysis is based on the theory of Alfvén wings: for a body immersed in a pulsar wind, a system of two stationary Alfvén waves is attached to the body, provided that the wind is highly magnetised. When they are destabilised through plasma instabilities, Alfvén wings can be the locus of strong radio sources that are convected with the pulsar wind. By assuming a cyclotron maser instability operating in the Alfvén wings, we make predictions about the shape, frequencies, and brightness of the resulting radio emissions.

Results. Because of the beaming by relativistic aberration, the signal is seen only when the companion is perfectly aligned between its parent pulsar and the observer, as is the case for occultations. For pulsar winds with a high Lorentz factor $\left(\geq 10^{4}\right)$, the whole duration of the radio event does not exceed a few seconds, and it is composed of one to four peaks that last a few milliseconds each and are detectable up to distances of several Mpc. The Lorimer burst, the three isolated pulses of PSR J1928+15, and the recently detected fast radio bursts are all compatible with our model. According to it, these transient signals should repeat periodically with the companion's orbital period.

Conclusions. The search of pulsar-orbiting bodies could be an exploration theme for new- or next-generation radio telescopes.
\end{abstract}

Key words. pulsars: general - radiation mechanisms: non-thermal - relativistic processes - minor planets, asteroids: general planet-star interactions

\section{Introduction}

Lorimer et al. (2007) discovered the first highly dispersed, bright radio burst in a Parkes pulsar survey. The time-frequency dispersion of this burst perfectly followed a $1 / f^{-2}$ law with a dispersion measure (DM) $\sim 375 \mathrm{pc} . \mathrm{cm}^{-3}$, out of which no more than $15 \%$ could be attributed to the wave path in our Galaxy. Therefore its origin was assumed to be extragalactic, with a source distance of about $500 \mathrm{Mpc}(z=0.12)$, well outside the local group of galaxies. As a consequence of this large distance and the strong burst intensity (30 Jansky $-1 \mathrm{Jy}=$ $\left.10^{-26} \mathrm{Wm}^{-2} \mathrm{~Hz}^{-1}\right)$, the inferred emitted power was extremely large, $\sim 10^{33} \mathrm{~J}$ (Thornton et al. 2013). The fixed-frequency duration of this burst is $\leq 5 \mathrm{~ms}$, with no detectable scatterbroadening, so that the natural origin of this exceptional burst was debated. But recently, five similar burtst (albeit less intense, 0.4-1.3 Jy) have been found in Parkes pulsar surveys (Keane et al. 2011; Thornton et al. 2013), and were called fast radio bursts (FRB). They emanate from different regions of the sky. Their fixed-frequency durations extend from shorter than $1.1 \mathrm{~ms}$ to $5.6 \mathrm{~ms}$. Interestingly, one of these bursts also exhibits an exponential scattering tail, supporting its natural origin. With DMs between $\sim 550$ and $1100 \mathrm{pc} \mathrm{cm}^{-3}$, all these bursts are attributed to extragalactic radio sources at cosmological distances in the range 1.7-3.2 Gpc. Their physical origin is unknown but again assumed to be extremely energetic. No event such as a supernova or a gamma-ray burst was observed simultaneously with an FRB. Proposed explanations include the annihilation of a mini black hole (Keane et al. 2012), binary white dwarf mergers (Kashiyama et al. 2013), neutron star mergers (Totani 2013), or implosions of supra-massive neutron stars shortly after their birth (Zhang 2014). All these explanations imply that FRBs are isolated events that occur only once from a given source. Other scenarii might lead to irregularly repeatable pulses, such as a giant pulse from a young pulsar with a low burst rate (Keane et al. 2012), or rare eruptions of flaring main-sequence stars within one kpc (Loeb et al. 2014).

We propose here a new explanation that does not require an extreme release of energy, but is still consistent with the observations. This explanation involves a body orbiting a pulsar: planet, asteroid, white dwarf, or even another neutron star. Many such objects are known in our Galaxy. Most millisecond pulsars are in binary systems, the companion being in many cases a white dwarf (Savonije 1987) - possibly of low mass (Bailes et al. 2011) - or a neutron star (Deller et al. 2013). Triple systems containing at least a pulsar and a white dwarf have been found 
(Ransom et al. 2014). Five planets, distributed within three planetary systems, are known to orbit pulsars (Wolszczan \& Frail 1992; Thorsett et al. 1993; Bailes et al. 2011). All of them were detected by analysing the shift of the pulsar period $P_{\mathrm{obs}}$ in terms of the position of the barycentre of the star, which led to the deduction that it was induced by the motion of orbiting planets. It is likely that other pulsar planets remain to be discovered. Finally, asteroid belts are likely to exist around pulsars, and they have been invoked by several authors to explain timing irregularities (Shannon et al. 2013), anti-glitches (Huang \& Geng 2014), or burst intermittency (Cordes \& Shannon 2008; Deneva et al. 2009; Mottez et al. 2013). In this paper we investigate the ability of these bodies immersed in a pulsar wind to produce radio waves.

Mottez \& Heyvaerts (2011b) showed that a pulsar-orbiting body necessarily moves in the wind of the pulsar and not in the light-cylinder, and that its interaction with the wind causes a system of strong electric currents flowing along the body and in the plasma carried by the wind. The power associated with these currents can be higher than $10^{22} \mathrm{~W}$. We explore what happens if a fraction of this power is converted into radio-frequency electromagnetic waves. Without needing to go into the detailed physics of the electromagnetic waves production, we can make predictions about the properties of the subsequent radio waves, and we study if and how this radio signal could be detected by ground-based radio-telescopes.

Our analysis is based on the electrodynamic interaction of the orbiting body and the pulsar wind derived in Mottez \& Heyvaerts (2011a,b) and Heyvaerts et al. (2012). This interaction is the relativistic analogue of the Io-Jupiter interaction, which has been described in terms of a unipolar inductor circuit or a pair of Alfvén wings (see the review by Saur et al. 2004) and transposed to exoplanet-star interaction by Zarka et al. (2001), Willes \& Wu (2005), and Zarka (2007). In our case, the magnetised plasma flow around the body is not corotating like in the previous papers, but is a wind as in Preusse et al. (2006), but here strongly relativistic.

In Sect. 2 we analyse the system of Alfvén wings generated by the interaction of the body with the pulsar wind. Then, after eliminating the possibility that the radio emission could come from the vicinity of the body (Sect. 3), we study the generation and characteristics of the radio emission produced by a windconvected source along the Alfvén wings (Sect. 4). In Sect. 5 we compare our predictions with past observations of a few remarkable radio transients. Finally in Sect. 6 we address specific questions raised by our explanation, and provide perspectives for observations that can confirm or refute our theory.

\section{Interaction with the pulsar wind}

A body orbiting a pulsar with a period exceeding a few minutes is immersed in the pulsar wind, and the interaction between the pulsar and its companion is ruled by electrodynamic processes. The distance of the known pulsar companions is typically several hundreds of light cylinder radii $\left(r_{\mathrm{LC}}\right)$.

Among the many pulsar wind models, those of Goldreich \& Julian (1969) and Michel (1969), or more recent ones (Bucciantini et al. 2006), show that for a pulsar with an aligned magnetic field, beyond a distance of hundreds of $r_{\mathrm{LC}}$ from the star, the plasma wind flow is radial $\left(v_{0} \sim v_{0}^{r}\right)$, the magnetic field energy dominates the plasma kinetic energy $\left(B^{2} \gg \mu_{0} \rho \gamma c^{2}-\right.$ the wind is said to be "Poynting-flux dominated"), and the plasma velocity is highly relativistic. The Lorentz factor is unknown but expected to be in the range $\gamma \sim 10^{1}-10^{7}$. The magnetic field is quasi-azimuthal $\left(B \sim B_{\phi} \gg B_{\text {poloidal }}\right)$. The flow and Alfvén velocities $\left(v_{0}\right.$ and $\left.V_{\mathrm{A}}\right)$ are very close to the speed of light and, if the above-quoted models are correct, the wind is sub-Alfvénic: $v_{0}<V_{\mathrm{A}}$ (Mottez \& Heyvaerts 2011b).

\subsection{Alfvén wings}

Because the fast magnetosonic waves are even faster than $V_{\mathrm{A}}$, the wind is also slower than the fast magnetosonic waves, and there is thus no MHD shock ahead of an obstacle in the wind. The pulsar-orbiting body is thus in direct contact with the wind. Following the idea developed in Neubauer (1980) and applied by Mottez \& Heyvaerts (2011b) in the context of pulsar winds, a pair of electric current circuits are induced by the body-wind interaction, carried by two stationary Alfvénic structures called Alfvén wings. These wings are attached to the body on one side, and extend far into space into the flowing plasma on the other side.

The azimuthal magnetic field of the pulsar wind is

$B_{0}^{\phi}=B_{0}^{r} \frac{v_{0}^{\phi}-\Omega_{*} r}{v_{0}^{r}} \sim-\frac{B_{0}^{r} \Omega_{*} r}{c}=-\frac{B_{*}^{r} \Omega_{*} R_{*}^{2}}{r c}$

where $B_{*}^{r}, \Omega_{*}$, and $R_{*}$ are respectively the star surface radial magnetic field, its rotation circular frequency, and radius. The convection electric field $E_{0}=v_{0}^{r} B_{0}^{\phi}=B_{0}^{r} \Omega_{*} r$, directed perpendicularly to the wind flow and to the magnetic field, is the engine of the Alfvén wings. It induces a potential drop $U=2 R_{\mathrm{b}} E_{0}$ along the body of radius $R_{\mathrm{b}}$, that is the cause of a current flowing along the planet and in the surrounding plasma.

When the Alfvén velocity is close to $c$, as in a pulsar wind, the total electric current is

$I_{\mathrm{A}} \sim 4\left(E_{0}-E_{\mathrm{i}}\right) R_{\mathrm{b}} / \mu_{0} c$,

where $E_{\mathrm{i}}$ is an electric field along the body caused by its ionospheric or surface finite conductivity. The power dissipated by Joule effect along the body is maximised when internal and external loads match, that is, when $E_{i}=E_{0} / 2$. For a pulsar with a rotation period of $1 \mathrm{~s}$ and an Earth-like orbiting body at $0.2 \mathrm{UA}, I_{\mathrm{A}} \sim 10^{11} \mathrm{~A}$. This current has the same order of magnitude as the current that powers the whole pulsar magnetosphere (Goldreich \& Julian 1969). For bodies orbiting recycled (millisecond) pulsars, the Alfvén wing current is smaller than the Goldreich \& Julian current by one or two orders of magnitude $\left(\sim 10^{9} \mathrm{~A}\right)$ because of a weaker wind magnetic field, but it is still not negligible.

For a pulsar whose magnetic dipole axis is aligned with the rotation axis and that has a radial wind flow and a mostly azimuthal magnetic field in the companion's environment, the angle $\delta$ between the invariant vector $\boldsymbol{V}_{s}$, i.e. the direction of the Alfvén wing, and the radial direction, can be estimated within simple models.

In most wind models for aligned pulsars (see Kirk et al. (2009) for a review), the wind is characterised by two invariants along its flow: the neutron star magnetic flux $\Psi$, and the mass flux $f$, defined as

$f=\gamma_{0} \rho_{0}^{\prime} v_{0}^{r} r^{2}$ and $\Psi=r^{2} B_{0}^{r}$,

with $\gamma_{0}$ the Lorentz factor associated with the unperturbed wind velocity $v_{0} \sim v_{0}^{r}$, and $B_{0}^{r}$ the radial magnetic field. Estimates of the Lorentz factor based on various observations and models vary over a fairly wide range from $10^{1}$ to $10^{7}$ (Kirk et al. 2009). 
Table 1. $x$ ratio, Afvén wing angles $\delta_{+}$and $\delta_{-}$with the radial wind direction, and proper time $\tau_{+}$and $\tau_{-}$spent by the CMI sources in the wings as functions of the companion distance $r$ and the wind Lorentz factor $\gamma_{0}$.

\begin{tabular}{lcccccc}
\hline \hline$r(\mathrm{AU})$ & $\gamma_{0}$ & $x$ & $\delta_{+}\left({ }^{\circ}\right)$ & $\delta_{-}\left({ }^{\circ}\right)$ & $\tau_{+} / \mathrm{s}$ & $\tau_{-}(\mathrm{s})$ \\
\hline 0.1 & 10 & 78. & 5.7 & -5.6 & $3 \times 10^{-3}$ & $3 \times 10^{-3}$ \\
0.01 & $10^{3}$ & $7.8 \times 10^{-2}$ & 1.4 & $-2 \times 10^{-3}$ & $1 \times 10^{-4}$ & $8 \times 10^{-2}$ \\
0.1 & $10^{3}$ & 0.78 & 0.16 & $-2 \times 10^{-2}$ & $1 \times 10^{-3}$ & $1 \times 10^{-2}$ \\
1.0 & $10^{3}$ & 7.83 & $6.5 \times 10^{-2}$ & $-5 \times 10^{-2}$ & $3 \times 10^{-3}$ & $4 \times 10^{-3}$ \\
40. & $10^{5}$ & 3.13 & $7.8 \times 10^{-4}$ & $-42 \times 10^{-4}$ & $2 \times 10^{-3}$ & $5 \times 10^{-3}$ \\
10. & $10^{5}$ & 0.78 & $1.7 \times 10^{-3}$ & $-2 \times 10^{-4}$ & $1 \times 10^{-3}$ & $1 \times 10^{-2}$ \\
0.1 & $10^{5}$ & $7.8 \times 10^{-3}$ & 0.14 & $-2 \times 10^{-6}$ & $\left(1 \times 10^{-5}\right)$ & 0.85 \\
0.01 & $10^{6}$ & $7.8 \times 10^{-5}$ & 1.4 & $-2 \times 10^{-9}$ & $\left(1 \times 10^{-7}\right)$ & 85 \\
\hline
\end{tabular}

Notes. A few values of $\tau_{+}$are in parenthesis because CMI is not expected with the $\delta_{+}$Alfvén wing when $x \ll 1$.

The angle $\delta_{s}$ of the Alfvén wings relative to the local magnetic field is

$\delta_{s}=s \arctan \left[\frac{x}{\gamma_{0}\left(\sqrt{1+x^{2}}-s\right)}\right]$,

where $s= \pm 1$, and $x$ is a dimensionless parameter defined by

$x=\frac{r}{\gamma_{0} r_{\mathrm{LC}}}$.

Details of this computation are given in Appendix A.

When $x \gg 1$, with Eq. (A.7) and $\gamma_{0} \gg 1$, Eq. (4) reduces to

$\delta_{s} \sim s \gamma_{0}^{-1}$.

This means that the two Alfvén wings make two symmetric and small angles relative to the radial direction. But we have no a priori evidence of the value of $x$. We might as well have $x \ll 1$. In that case, the two Alfvén wings have an asymmetric configuration with an angle $\delta_{-}=-x / 2 \gamma_{0}=r_{\mathrm{LC}} /\left(2 \gamma_{0}^{2} r\right)$ even smaller than in the previous case, and an angle $\delta_{+}=\arctan 2 / \gamma_{0} x=2 r_{\mathrm{LC}} / r$.

A few examples of $\delta_{ \pm}$angles are displayed in Table 1 for various values of $r$ and $\gamma_{0}$.

We can consider two types of sources of radio-emissions: the source can be (1) attached to the companion body, or more precisely, to a plasma with a low velocity in the body's frame of reference; or it can be (2) carried along with the pulsar wind plasma.

\section{Radio emission from a source linked to the companion}

The plasma close to the pulsar companion can be destabilised by the Alfvén wing's current flowing along the body, and excite a high level of coherent electromagnetic waves. Then, in the reference frame of the companion, the radio emission may be sent at various angles, as is observed near solar system planets connected to Alfvén wings. For instance, Jupiter's Galilean satellite Io is embedded in the co-rotating magnetosphere of Jupiter and is the source of two Alfvén wings (Neubauer 1980; Saur et al. 2004).

The wings in the vicinity of Jupiter are strong radio emitters of decametric waves that are observed with ground-based radiotelescopes. Their frequencies cover the range of local electron cyclotron frequencies in the source regions. It is not clear if the sources are fixed relative to the Alfvén wings or if they are convected by the co-rotating plasma, but in both cases, their velocity would be too low to be measured by Doppler shift.
For a pulsar companion, sources of radio waves attached to the companion or to the co-rotating plasma in its vicinity would also have a non-relativistic velocity relative to the observer. The relativistic aberration would be negligible as well and would not cause any focussing of the emitted energy. Therefore, even if the source is powerful, these radio waves would be very faint at interstellar distances and the chances to detect them from Earth would be very low. As a comparison, the intense Io-Jupiter radio emission is not detectable against the Galactic background beyond $1 \mathrm{pc}$ distance even with the largest existing radio-telescopes (Farrell et al. 2004; Zarka 2004a). Detection at distances of the order of a kpc would thus require an emission $>10^{6}$ times more intense.

Because their emission would not be detectable from Earth, we did not investigate the assumption of radio sources attached to the companion further.

\section{Sources of radio emission carried by the wind along the wings}

Radio emissions might also be emitted from the plasma wind when it crosses the Alfvén wings, far from the pulsar companion. The sources would be convected with the wind along the Alfvén wings. Because the wind is relativistic, the sources would have a relativistic motion relative to an observer on Earth. More precisely, we assume that the source propagates along the Alfvén wing, and that the source velocity projected along the (radial) wind direction is the same as that of the wind. Then, the source velocity modulus is $V_{s}=v_{0} / \cos \delta \sim v_{0}\left(1+\sin \delta^{2}\right)^{1 / 2}=c^{2}(1-$ $\left.\gamma_{0}^{-2}\right)\left(1+\delta^{2}\right)$. The requirement that $V_{s}<c$ implies $\delta^{2}<\left(\gamma_{0}^{2}-1\right)^{-1}$. It is satisfied with the two wings if $x \gg 1$, and with the $\delta_{-}$wing only when $x \ll 1$. Thus, at least one source can always exist.

\subsection{Plasma flow, instability, and radio waves}

We define the observer's frame in the Cartesian coordinate system where $O z$ is aligned with the Alfvén wing velocity $\boldsymbol{V}_{s}$ and where the magnetic field is contained in the plane $x \mathrm{Oz}$ (cf. Fig. 1). The source frame has the same axes, but moves at velocity $\boldsymbol{V}_{s}=V_{s} \boldsymbol{e}_{z}$ along $O z$. The pulsar wind velocity in the observer's frame is $\left(v_{0} \sin \delta, 0, v_{0} \cos \delta\right)$. The wind velocity in the source frame, noted $\boldsymbol{v}^{\prime}$, is given by

$v_{z}^{\prime}=\frac{v_{0} \cos \delta-V_{s}}{1-\frac{V_{s}^{2} \cos \delta}{c^{2}}}$ and $v_{x}^{\prime}=\gamma v_{0} \sin \delta\left[1+\frac{V_{s}}{c^{2}} v_{z}^{\prime}\right]$, 


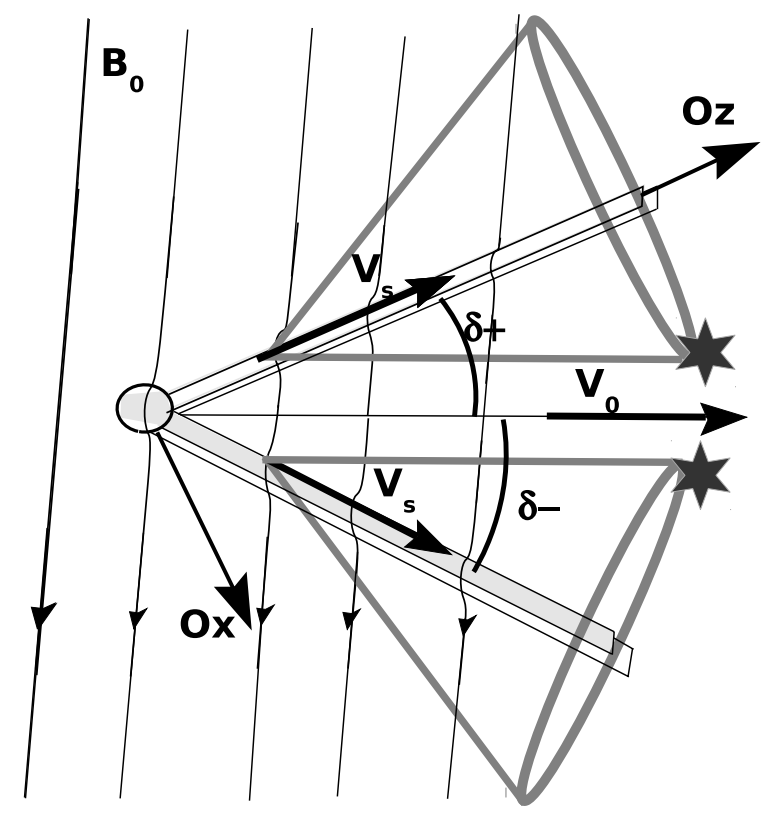

Fig. 1. Alfvénic wake of the pulsar's companion body seen from above the equatorial plane. The velocity of the source regions $\boldsymbol{V}_{s}$ is assumed to be along the Alfvén wings. The thick grey lines represent the cones in which emitted radio waves are focussed. We call them the angular emission patterns. The observer's frame related to the upper wing on the figure (making an angle $\delta_{+}$with the wind) is indicated by the $O x$ and $O z$ axes. It is fixed relative to the pulsar companion. The two stars mark the region of the angular emission patterns of highest wave intensity (see Sect. 4).

where $\gamma=\left(1-V_{s}^{2} / c^{2}\right)^{-1 / 2}=\left(1-v_{0}^{2}\left(1+\sin ^{2} \delta\right) / c^{2}\right)^{-1 / 2} \sim \gamma_{0}$. If the source propagates with the wind, as assumed here, then $V_{s}=$ $v_{0} \cos \delta, v_{z}^{\prime}=0, v_{x}^{\prime}=v_{0} \gamma \delta=v_{0} s x /\left(\sqrt{x^{2}+1}-s\right)$ is a significant fraction of $v_{0}$. While in the observer's frame the magnetic field is mostly azimuthal $\left(B_{0}^{\phi} \gg B_{0}^{r}\right)$, this is not true in the source frame. In that frame the magnetic field is

$B_{s}^{r}=B_{0}^{r}$ and $B_{s}^{\phi}=\frac{B_{0}^{\phi}}{\gamma} \ll B_{0}^{\phi}$,

thus a large component parallel to the wing exists, as illustrated in Fig. 2.

In terms of particle distribution (a Lorentz invariant), $f(\boldsymbol{r}, \gamma m \boldsymbol{v})=f\left(\boldsymbol{r}^{\prime}, \gamma m \boldsymbol{v}^{\prime}\right)$, so that a distribution that is shifted in the $v_{z}$ direction in the observer's frame is shifted in the $v_{x}^{\prime}$ direction in the source frame. The particle distribution is then highly non-gyrotropic, since $O x$ is a direction that creates a large angle with the magnetic field in the source frame. This shifted distribution is expected to be a powerful energy source for the cyclotron maser instability (CMI; Freund et al. 1983; Wu 1985). We note that the CMI theory is based on special relativity effects, even when applied in the midly relativistic plasma of main-sequence stars and in the Io Alfvén wings. The CMI does not exist in a non-relativistic plasma; it requires a particle population with non-negligible $v / c$.

We did not compute the CMI growth rate here. It would require to know the precise shape of the particle distribution function, for which we have no measurement or theoretical arguments that would justify any choice at this stage. Nevertheless, the crucial part of the CMI theory concerns the cyclotron resonance condition. It is developed in the relativistic context without any approximation of the Lorentz factor (Wu 1985). In that

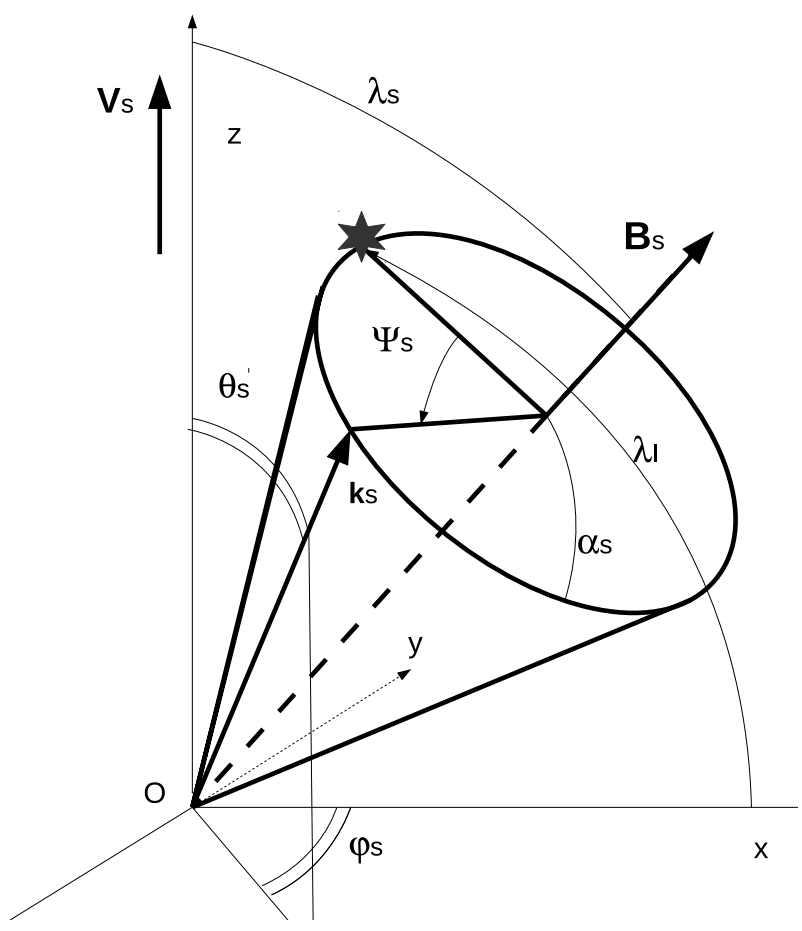

Fig. 2. Directions of the magnetic field and of the radio waves emitted in the source frame: the wave vectors are along a cone of summit angle $\alpha_{s}$, and of an axis parallel to the magnetic field $\boldsymbol{B}_{s}$. The wave vector position on that cone is parametrised by the angle $\Psi_{s}$. The inclination of the magnetic field with the wing axis $O z$ is $\lambda_{s}$. The cylindrical angular coordinates $\theta_{s}$ and $\varphi_{s}$ of the wave vector are defined relatively to the $z$ axis. As in Fig. 1, the star (at $\left.\Psi_{s}=0\right)$ marks the angle of emission of the most intense CMI waves.

general context, the most unstable waves have the following properties in the reference frame of the source:

- The wave vectors are aligned along a cone of summit angle $\alpha_{s}$ whose axis is the magnetic field in the source reference frame.

- Because the average velocity of the distribution is low in the direction of the magnetic field, the wave vectors are expected to make a large angle with the magnetic field. We can consider that the unstable wave vectors lie along a cone of aperture half-angle $\alpha_{s}$ in the range $85^{\circ}-90^{\circ}$.

- The growth rate does not depend on the azimuth $\Psi_{s}$ of the wave vector along the cone if the distribution function is gyrotropic (i.e. of the form $f=f\left(\boldsymbol{r}, p_{\|}, p_{\perp}\right)$ ). In contrast, the growth rate can depend strongly on $\Psi_{s}$ if $f$ explicitly depends on the three components of $\boldsymbol{p}$. In the present case, as shown above, the distribution is non-gyrotropic, and the intensities of the waves should depend on $\Psi_{s}$, the most intense waves on this cone corresponding to wave vectors $\boldsymbol{k}$ in the $x$ direction, mostly in the direction of the wind $(+x$ for the $\delta+$ wing), and possibly also in the opposite direction.

- The frequency of the emitted waves is close to the local electron gyrofrequency $\omega \simeq \omega_{\text {ce }}$ in the linear approximation $-x$ for the $\delta+$ wing.

- When the growth rate is high, non-linear effects tend to produce harmonics and broaden the emitted frequency spectrum.

How much time does an element of plasma of the wind spend in the Alfvén wing? We assume that the diameter of the Alfvén wing is $\eta R_{\mathrm{b}}$ where $\eta$ is of the order of one. The inclination angle 
of the wind relative to the Alfvén wing being $\delta=1 / \gamma$, an element of plasma of the wind spends a time $\tau_{\mathrm{o}} \sim \eta R_{\mathrm{b}} / \delta v_{0}$ in the wing, in the observer's reference frame. In the reference frame of the source, this time is

$\tau_{s} \sim \eta R_{\mathrm{b}} / \delta \gamma_{0} v_{0}$

A few values of $\tau_{s}$ are displayed in Table 1 , where it is assumed that $\eta R_{\mathrm{b}}=1000 \mathrm{~km}$, and $v_{0} \sim c$. We can see that these times currently exceed a few milliseconds, especially in the $\delta_{-}$wing. This is much longer than the typical growth time of the CMI, which can reach saturation in tens to hundreds of microseconds in much less anisotropic and energetic plasmas (Pritchett 1986; Le Queau \& Roux 1987; Le Queau 1988).

\subsection{Direction of radio waves emitted by the cyclotron maser instability}

Let $\theta_{s}$ and $\varphi_{s}$ define the direction of the electromagnetic wave vector in the source frame (Fig. 2). Because of the relativistic aberration, these angles become in the observer's frame:

$$
\cos \theta_{\mathrm{o}}=\frac{\cos \theta_{s}+\beta}{1+\beta \cos \theta_{s}} \text { and } \varphi_{\mathrm{o}}=\varphi_{s}
$$

where $\beta=V_{s} / c$. A well known consequence of Eq. (10) is that for high values of $\gamma$ and for any radiation angle $\theta_{s}>0$, the corresponding angles $\theta_{0}$ in the observer's frame are smaller than $\gamma^{-1}$. We will show that this is correct provided that $\gamma \gg 10^{3}$. The luminosity of the emission is also affected by the aberration. Let $\mathrm{d} W_{\mathrm{o}} / \mathrm{d} \Omega_{\mathrm{o}}$ be the energy radiated in the solid angle $\Omega_{\mathrm{o}}$ in the observer's reference frame, and $\mathrm{d} W_{s} / \mathrm{d} \Omega_{s}$ the same ratio in the source frame, then

$$
\frac{\mathrm{d} W_{\mathrm{o}}}{\mathrm{d} \Omega_{\mathrm{o}}}=\frac{\mathrm{d} W_{s}}{\mathrm{~d} \Omega_{s}} \frac{\left(1-\beta^{2}\right)^{2}}{\left(1-\beta \cos \theta_{\mathrm{o}}\right)^{3}}=\frac{\mathrm{d} W_{s}}{\mathrm{~d} \Omega_{s}} \gamma^{2}\left(1+\beta \cos \theta_{s}\right)^{3} .
$$

For an isotropic radiation in the source frame, $\frac{\mathrm{d} W_{s}}{\mathrm{~d} \Omega_{s}}$ is constant and the main effect on $\frac{d W_{0}}{d \Omega_{0}}$ is the amplification by $\sim \gamma^{2}$ resulting from the transformation of the solid angle of emission from the source frame to the observer's frame. For instance, all the radiation for $\theta_{s}>0$ is radiated in the observer's frame in the solid angle $\mathrm{d} \Omega_{\mathrm{o}} \sim$ $\pi \gamma^{-2}$ sr delimited by the cone of summit angle $\gamma^{-1}$, which is very small if $\gamma \gg 1$. Conversely, all the radiation for $\theta_{s}<0$ is emitted in the other directions corresponding to a solid angle $\Omega=\pi(4-$ $\left.\gamma^{-2}\right)$ sr. Therefore, the power angular density $\mathrm{d} W_{\mathrm{o}} / \mathrm{d} \Omega_{\mathrm{o}}$ is much higher inside the cone of summit angle $\gamma^{-1}$ than in the other directions. That is why we can say that most of the observable radiation is contained within the cone of angle $\gamma^{-1}$. If $\gamma \gg 1$, the amplitude of the signal is lower by orders of magnitude in the other directions.

Theories of the CMI tell us, however, that the radio waves are not emitted isotropically, but rather along conical sheets of aperture angle $\alpha_{s}$ varying with the frequency in a range close to $90^{\circ}$. If the particle distribution is non-gyrotropic, the intensity of the waves can be highly variable along the cone walls.

We first consider the direction of the wave vectors associated with a single Alfvén wing aligned with $O z$. We consider waves whose vectors $\boldsymbol{k}$ form a cone of angle $\alpha_{s}$ with the magnetic field in the source reference frame. What are the wave directions in the observer's reference frame? Let $\lambda_{s}$ be the angle between the emission cone axis and the $z$ direction in the source frame (Fig. 2). Because the emission cone axis is parallel to the magnetic field, $\tan \lambda_{s}=r / \gamma r_{\mathrm{LC}}$. A single wave vector is characterised by the cone of axis $\boldsymbol{B} / \boldsymbol{B}$, of summit angle $\alpha_{s}$, and by its position along the cone, parametrised by the angle $\Psi_{s}$. This wave vector direction is characterised by the two angles $\theta_{s}$ and $\varphi_{s}$ in the spherical coordinates frame associated with the $O z$ axis in the source frame. The coordinates of the unit vector parallel to the wave vector are

$$
\begin{aligned}
k_{x}= & \cos \varphi_{s} \sin \theta_{s}=\cos \lambda_{I} \cos \Psi_{s} \\
& +\frac{r_{n}}{1+r_{n}^{2}}\left(r_{n} \cos \lambda_{I}+\sin \lambda_{I}\right)\left(1-\cos \Psi_{s}\right), \\
k_{y}= & \sin \varphi_{s} \sin \theta_{s}=\frac{1}{\sqrt{1+r_{n}^{2}}}\left(\cos \lambda_{I}-r_{n} \sin \lambda_{I}\right) \sin \Psi_{s}, \\
k_{z}= & \cos \theta_{s}=\sin \lambda_{I} \cos \Psi_{s} \\
& +\frac{1}{1+r_{n}^{2}}\left(r_{n} \cos \lambda_{I}+\sin \lambda_{I}\right)\left(1-\cos \Psi_{s}\right),
\end{aligned}
$$

where $\lambda_{I}=\pi / 2-\lambda_{s}+\alpha_{s}$ and $r_{n}=r / \gamma r_{\mathrm{LC}}=\tan \lambda_{s}$. The direction angles $\theta_{s}$ and $\varphi_{s}$ can be easily derived and expressed in the observer's frame following Eq. (10). When $\beta$ is close to one, it is better to use the following relation which resolves a problem of numerical degeneracy,

$\theta_{\mathrm{o}}=\frac{1}{\gamma} \sqrt{\frac{1-\cos \theta_{s}}{1+\beta \cos \theta_{s}}}$.

The above angles are relative to a single Alfvén wing, and the $z$ axis corresponds to the wing direction.

As mentioned, the luminosity is expected to be much higher in the direction corresponding to $\Psi_{s}=0$.

When the two wings are considered, it is better to define a $Z$ axis along the wind direction, and to shift the above values of $\theta_{0}$ (related to a wing $z$ axis) by an angle $\delta_{-}$for one wing and $\delta_{+}$ for the other. The directions of the waves are plotted in Fig. 3 in the observer's frame for a planet at $0.1 \mathrm{AU}$ from the star, an emission angle $\alpha_{s}=85^{\circ}$, and $\gamma=10$ (the low range of $\gamma$ for a pulsar wind). The small grey disks represent the field illuminated by waves emitted at an angle $\leq 90^{\circ}$ from each Alfvén wing axis in the source frame (cf. Fig. 2) in the approximation of very large $\gamma$. There is one such disk for each Alfvén wing, whose border corresponds to emission at $90^{\circ}$ from the Alfvén wing axis in the source frame. These circles are the same as those that limit the cones represented on Fig. 1. Because these grey disks do not encompass the other circles, the approximation of a very large $\gamma$ is not valid when $\gamma=10$.

The angular emission patterns can be related to time intervals. We first neglect the oscillation of the (tilted) magnetic field with the pulsar spin frequency. The simplest trajectory of the line of sight to the observer on these figures is a straight line uniformly parametrised in time, deduced from the orbital motion of the pulsar companion. In Fig. 3, there would be four intersections of the emission beam (large circles) with this straight line, two of which are associated with a high-amplitude signal. An angle interval $\Delta$ corresponds to a time interval $\tau$ given by

$\left(\frac{\mathrm{deg}}{\Delta}\right)\left(\frac{\tau}{\mathrm{s}}\right)=240\left(\frac{T_{\text {orb }}}{\text { day }}\right)$.

When the oscillation of the magnetic field is taken into account, the whole figure rotates back and forth around the origin at the pulsar period with an angular amplitude depending mainly on the pulsar tilt angle. Then, provided that the time $\tau$ is longer than the pulsar spin period, we can have several pulses for each crossing of the beam pattern instead of one longer pulse when the oscillation is negligible. 


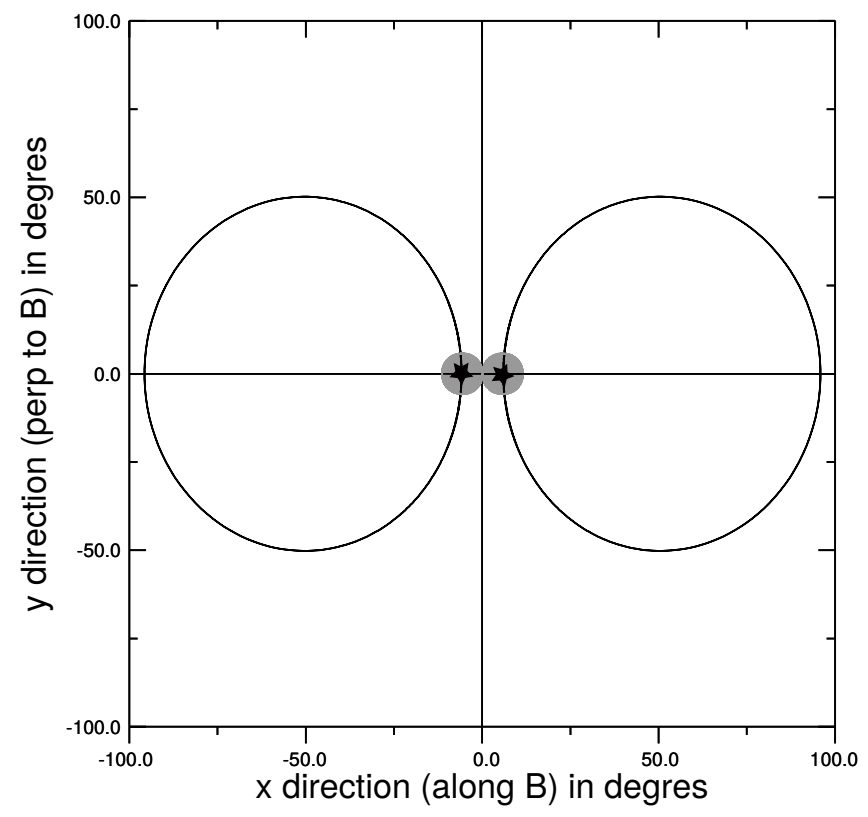

Fig. 3. Directions of the radio waves in the observer's frame. The two smaller tangent grey disks in the middle of the plot correspond to the waves that are beamed at $\leq 90^{\circ}$ from the Alfvén wing axis in the source frame in the approximation of very high $\gamma$ values (see text for the precise meaning of "very high"). The two large circles (black lines) are the radio-beaming patterns associated with the CMI. They are defined by $x=\delta_{ \pm}+\theta_{0} \cos \varphi_{0}$ and $y=\theta_{0} \sin \varphi_{0}$, with $\theta_{0}$ and $\varphi_{0}$ the cylindrical angular coordinates of the wave vector in the observer's reference frame of each Alfvén wing. $\theta_{\mathrm{o}}$ and $\varphi_{\mathrm{o}}$ are related via Eq. (10) (or Eq. (13)) to the wave vector coordinates in the source frame $\theta_{s}$ and $\varphi_{s}$ given by Eq. (12). The most intense emissions correspond to the innermost intersection of each large circle with the horizontal axis. Here $\gamma=10, r=0.1 \mathrm{AU}$ and $\alpha_{s}=85^{\circ}$. With these parameters, $x=78$. The stars mark the directions of the most intense radio emissions.

For $\gamma=10$, the angle $\lambda_{s}$ between the cone axis and $O z$ in the source frame is large, so that a large part of the emission cone of summit angle $85^{\circ}$ is beamed at $>90^{\circ}$ from $\mathrm{Oz}$. In the observer's frame, we see in Fig. 3 that these radio emissions make a large angle - of tens of degrees - with the wind direction. Because of the orbital motion of the pulsar companion, a distant observer will cut the emission diagram along a horizontal line shifted from the horizontal axis of Fig. 3 by the inclination of the companion's orbital plane as seen from Earth. If the pulsar magnetic field is tilted on its rotation axis, then the beaming pattern will oscillate at the pulsar spin period (and thus the observer will cut the emission diagram along a sinusoidal curve). In all cases, the observer probably intersects the radio emission diagram four times during a full companion's orbit. Because of the relativistic transformation of solid angles and because $\Psi_{s}=0$ corresponds to much more powerful emissions than in the other directions, however, the most intense emissions will be seen at the crossings of the radio-beaming diagram close to the origin, inside the grey disks.

The situation is quite different for $\gamma=1000$, as shown in Fig. 4. The range of angles of emission is now much smaller than $1^{\circ}$. Therefore, the chances of being in the correct line of sight are much lower. If it is so, the observer can see the radio emission up to four times (out of which two correspond to intense emission) that are much closer in time to each other. At a distance of $0.1 \mathrm{AU}$ from a pulsar with a mass of $1.4 M_{\odot}$, the orbital period is $\sim 10$ days, and the typical separation of $0.16^{\circ}$ between the two innermost intersections of the radio-beaming

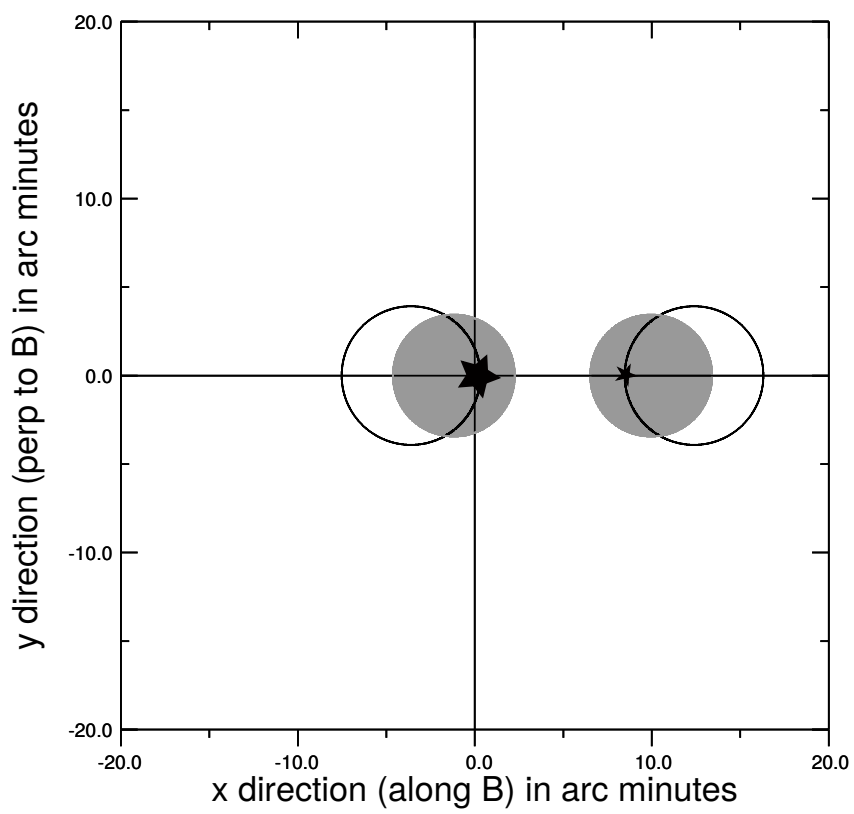

Fig. 4. Directions of the radio waves in the observer's frame. Same as in Fig. 3, but for $\gamma=1000$. As in Fig. 3, $r=0.1 \mathrm{AU}$ and $\alpha_{s}=85^{\circ}$. These parameters correspond to $x=0.78$.

pattern correspond to time intervals of $\sim 6$ minutes. Because of the already mentioned anisotropy of the distribution function, the two inner regions (near the two inner intersections with the horizontal line) correspond to much higher amplitudes than the two others.

Only the emission pattern associated with $\delta_{-}$is shown in Fig. 5 for $\gamma=10^{6}$. As we can see in Table 1 , the time spent by the CMI sources in the wing $\delta_{-}$is $85 \mathrm{~s}$, while it is about $0.1 \mu \mathrm{s}$ in the other wing. Furthermore, CMI source cannot propagate at the wind velocity along the $\delta_{+}$wing when $x \ll 1$. The directions of emission associated with the $\delta_{-}$wing almost coincide with the tangent circle that limits the grey disk (they coincide exactly for $\alpha_{s}=90^{\circ}$ ) and the corresponding angles are now of the order of $0.2^{\prime \prime}$. We chose a distance $r=0.01 \mathrm{AU}$, corresponding to $T_{\text {orb }} \sim 9 \mathrm{~h}$ (with a neutron star of 1.4 solar mass). According to Eq. (14), the time interval between two circle crossings is shorter than $5 \mathrm{~ms}$. The vicinity of the intersection of the circle with the horizontal line, on the right-hand side, corresponds to the highest wave amplitude.

For $\gamma=10^{5}$ and $r=0.1 \mathrm{UA}$, the figure (not shown) has the same aspect as in Fig. 5, with an anglular diameter $\sim 2$ " instead of $0.2^{\prime \prime}$. This corresponds to a time interval of $1.3 \mathrm{~s}$.

Because most pulsar wind models are based on highly relativistic flows, and because, as we show below, it may explain some already existing observations, we focus the following analysis on the cases where $\gamma>10^{3}$.

\subsection{Range of frequencies}

As mentioned in Sect. 4.1, we first considered that the waves are emitted at frequencies close to the local gyrofrequency $f_{\mathrm{c}, \mathrm{s}}$ in the reference frame of the sources. Then we converted the frequency to $f_{\mathrm{c}, \mathrm{o}}$ into the frame of the observer. Using Eqs. (1) and (8) and considering that $\Psi=B_{*} R_{*}^{2}$, the gyrofrequency in the source frame is

$f_{\mathrm{c}, \mathrm{s}}=\frac{q B_{*}}{2 \pi m} \frac{R_{*}^{2}}{r^{2}} \sqrt{1+\left(\frac{r}{\gamma r_{\mathrm{LC}}}\right)^{2}}$, 
F. Mottez and P. Zarka: Transient radio emissions from pulsar-orbiting bodies

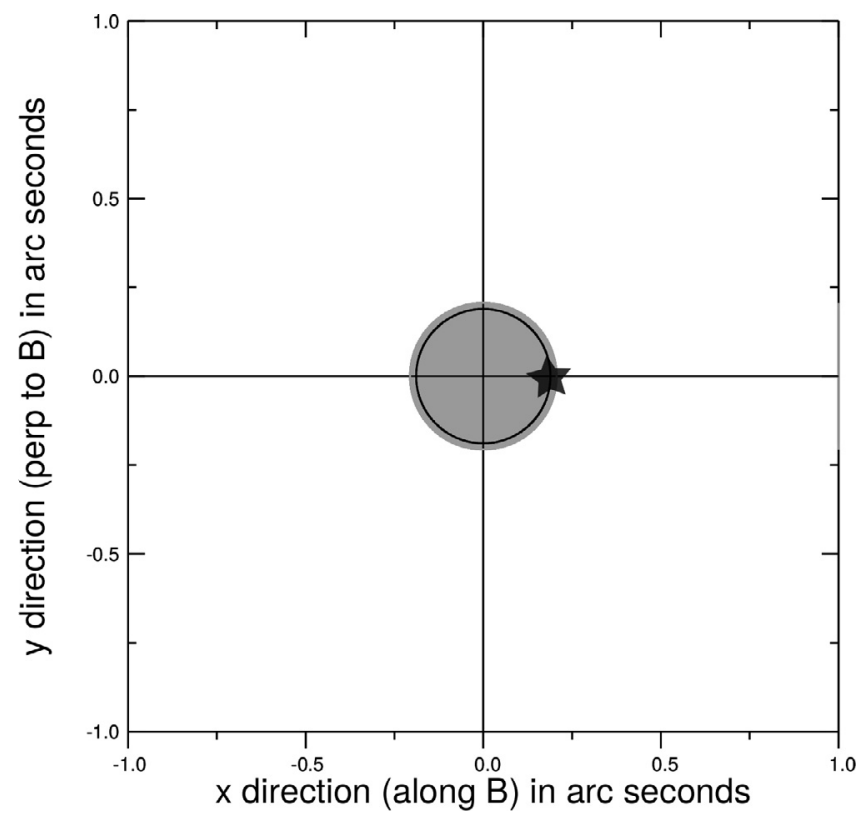

Fig. 5. Directions of the radio waves in the observer's frame. Same as in Fig. 3, but for $\gamma=10^{6}$ and $r=0.01$ AU. As in Fig. 3, $\alpha_{s}=85^{\circ}$. These parameters correspond to $x=0.78 \times 10^{-5}$. Here only the emission pattern associated with $\delta_{-}$is represented. The star marks the direction of the most intense radio emissions.

with $q$ and $m$ the electron's charge and mass. The DopplerFizeau shift along the line of sight towards the observer provides the observed frequency

$$
\begin{aligned}
f_{\mathrm{c}, \mathrm{o}}= & 25 \gamma\left(\frac{B_{*}}{10^{5} \mathrm{~T}}\right)\left(\frac{1 \mathrm{AU}}{r}\right)^{2}\left(\frac{R_{*}}{10^{4} \mathrm{~m}}\right)^{2} \\
& \times\left\{1+\left[\frac{\pi 10^{5}}{\gamma}\left(\frac{10 \mathrm{~ms}}{T_{*}}\right)\left(\frac{r}{1 \mathrm{AU}}\right)\right]^{2}\right\}^{1 / 2} .
\end{aligned}
$$

Figure 6 displays the observed frequency $f_{\mathrm{c}, \mathrm{o}}$ as a function of the Lorentz factor $\gamma$ of the pulsar wind for various companion distances from a millisecond pulsar. For $\gamma<10^{5}$ the frequencies are in the range of meter to decameter radio waves, typically reachable with LOFAR (van Haarlem et al. 2013) and (in the future) $\mathrm{SKA}^{1}$. The $\mathrm{GHz}$ range of frequencies is reached for companions within $0.2 \mathrm{AU}$ when the Lorentz factor is higher than $10^{6}$. The sub-millimeter range is reached only for very close planets and very fast pulsar winds $\left(\gamma>10^{7}\right)$.

Figure 7 displays the observed frequency/Lorentz factor relation for a standard pulsar of period $T_{*}=1 \mathrm{~s}$ and magnetic field $B_{*}=10^{8} \mathrm{~T}$. Up to now, no planet is known to orbit a standard pulsar, but other companions are likely to exist. For such systems, observed frequencies will be significantly higher than those associated with millisecond pulsar companions. They will remain in the radio range for distant companions, but could reach the infrared domain for close-by companions and high-speed winds.

\subsection{Brightness}

The maximum power involved in an Alfvén wing is (Mottez \& Heyvaerts 2011b)

$\dot{E}_{J}=\frac{\pi c}{\mu_{0}} R_{\mathrm{b}}^{2}\left(B_{0}^{\phi}\right)^{2}$.

\footnotetext{
1 http://www. skatelescope.org
}

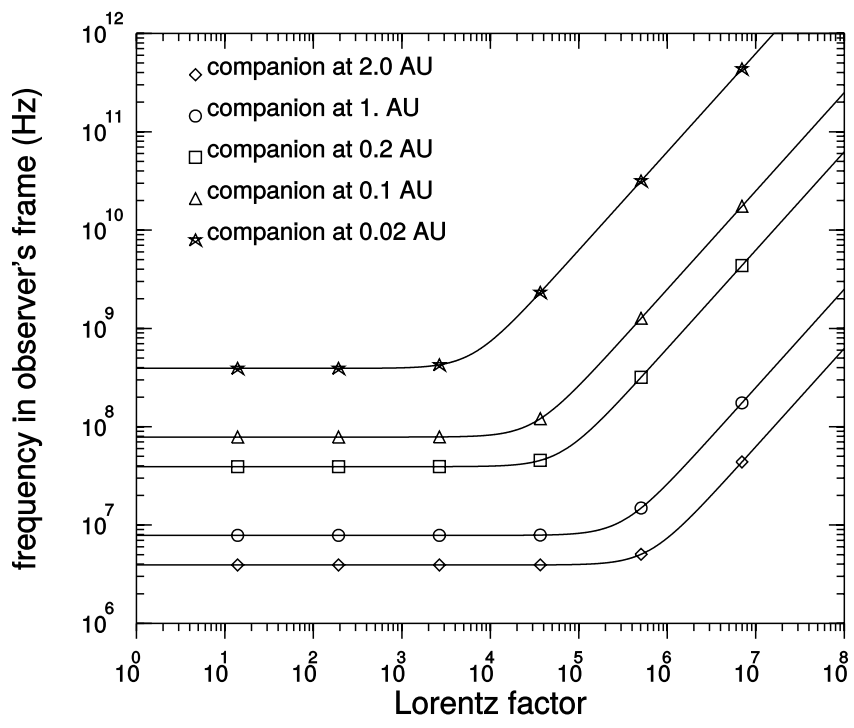

Fig. 6. Cyclotron frequency in the observer's frame as a function of the pulsar wind Lorentz factor $\gamma$, from Eq. (16) for various distances of the companion. Values that are not varied are the same as in the text: the magnetic field is $B_{*}=10^{5} \mathrm{~T}$ and the spin period $T_{*}=10 \mathrm{~ms}$.

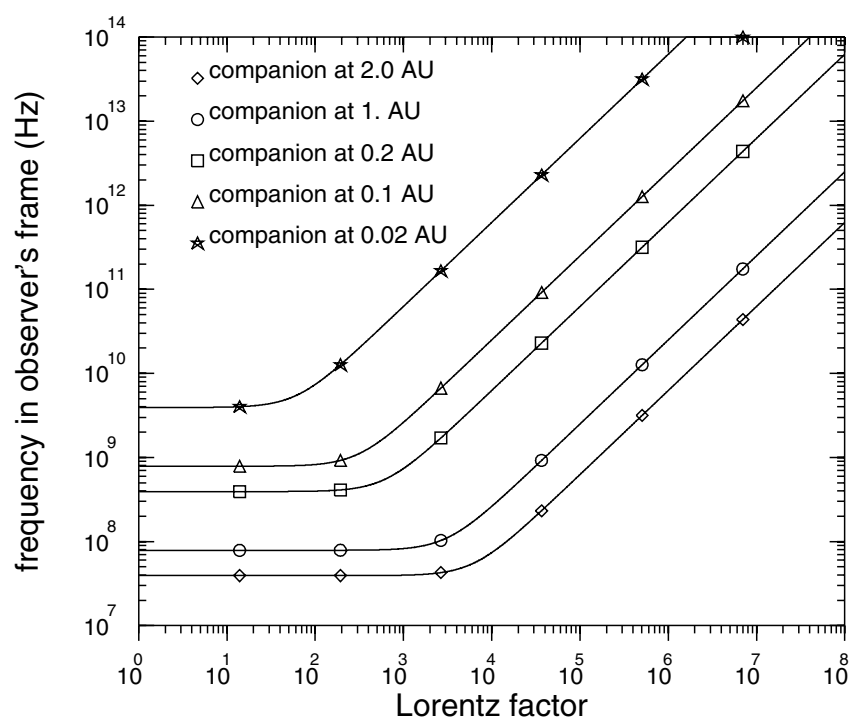

Fig. 7. Same as Fig. 6 for companions orbiting a standard pulsar with a magnetic field $B_{*}=10^{8} \mathrm{~T}$ and a spin period $T_{*}=1 \mathrm{~s}$.

This expression is the relativistic analogue of that derived in Zarka et al. (2001) for magnetised flow-obstacle interactions. From Eq. (1), this can be expressed as a function of the distance $r$ and the radius $R_{\mathrm{b}}$ of the orbiting body

$\dot{E}_{J}=\frac{\pi}{\mu_{0} c} R_{\mathrm{b}}^{2} r^{-2} R_{*}^{4} B_{*}^{2} \Omega_{*}^{2}$.

From studies of planetary magnetospheric radio emissions in our solar system, it was found that the emitted radio power is proportional to the Poynting flux of the solar wind on the magnetospheric cross-section. The same relation of proportionality also relates satellite-induced (Io, Ganymede) radio emissions in the magnetosphere of Jupiter with the incident Poynting flux convected by the rotation of the planetary magnetic field on the obstacle (ionosphere of Io, magnetosphere of Ganymede). The general scaling law describing this relation has a constant of proportionality $\epsilon \sim 2-10 \times 10^{-3}$ (Zarka et al. 2001; Zarka 2007). 
To test the possible saturation of this scaling law toward high Poynting fluxes and high radio powers, Zarka (2010) analysed the literature on radio emission from magnetised binary stars. Budding et al. (1998) and Richards et al. (2003) showed that the RS CVn system V711 $\tau$ frequently emits radio bursts that reach $0.1 \mathrm{Jy}$ at $2.3 \mathrm{GHz}$ and $1 \mathrm{Jy}$ at $8.4 \mathrm{GHz}$. With a distance of $29 \mathrm{pc}$ and a bandwidth $\Delta f \geq 8 \mathrm{GHz}$, one deduces an isotropic radio power $\sim 7 \times 10^{19-20} \mathrm{~W}$. Based on estimates from Saar (1996) and Budding et al. (1998), the RS CVn magnetic fields reach $1-3 \times 10^{3} \mathrm{G}$, which leads, based on the separation of the system V711 $\tau\left(11.5 R_{\odot}\right.$, Richards et al. 2003), to a magnetic field magnitude $B \sim 10-30 \mathrm{G}$ at the interaction region. The radius of this region is at most that of the secondary companion in V711 $\tau$ $\left(\sim 1.3 R_{\odot}\right)$. Assuming a stellar wind velocity $V \sim 300 \mathrm{~km} \mathrm{~s}^{-1}$ and an interaction region size $R \sim 1-9 \times 10^{8} \mathrm{~m}$, the Poyinting flux convected on the obstacle is $\frac{V B^{2}}{\mu_{0}} \pi R^{2}=7 \times 10^{21}-6 \times 10^{24} \mathrm{~W}$. The constant of proportionality $\epsilon$ is thus in the range $10^{-1}$ to $10^{-5}$, but more reasonably (matching high and low estimates) in the range $10^{-2}$ to $10^{-4}$. In spite of the above uncertainties, the range of $\epsilon$ falls remarkably close to that deduced from the above scaling law, suggesting that it may remain valid with a constant of about a few $10^{-3}$ up to 10 orders of magnitude above the range of solar system planets.

Therefore, we estimate that the power radiated in the form of non-thermal radio waves is in our case

$P_{\text {radio }}=\epsilon \dot{E}_{J}=\epsilon \frac{\pi}{\mu_{0} c} R_{\mathrm{b}}{ }^{2} r^{-2} R_{*}^{4} B_{*}^{2} \Omega_{*}^{2}$,

with a conservative value of about $10^{-3}$ for $\epsilon$.

For a spectrum of emitted waves spreading along an interval $\Delta f$ of frequencies, at a distance $D$ from the source, the average flux density in Jy of radio waves inside the cone of emission is

$$
\begin{aligned}
\left(\frac{\langle S\rangle}{\mathrm{Jy}}\right)= & 6.5\left(\frac{\gamma}{10^{5}}\right)^{2}\left(\frac{\epsilon}{10^{-3}}\right)\left(\frac{R_{\mathrm{b}}}{10^{7} \mathrm{~m}}\right)^{2}\left(\frac{1 \mathrm{AU}}{r}\right)^{2}\left(\frac{R_{*}}{10^{4} \mathrm{~m}}\right)^{4} \\
& \times\left(\frac{B_{*}}{10^{5} \mathrm{~T}}\right)^{2}\left(\frac{10 \mathrm{~ms}}{T_{*}}\right)^{2}\left(\frac{\mathrm{Mpc}}{D}\right)^{2}\left(\frac{1 \mathrm{GHz}}{\Delta f}\right) .
\end{aligned}
$$

The detected flux density will be $S=\langle S\rangle$ only if the radiation is isotropic in the source frame. If this is not the case, an additional amplification factor intrinsic to the emission mechanism is to be applied for specific directions of emissions inside the cone, whereas $S$ can be null outside of these specific directions.

For the reference values of Eq. (20), Fig. 8 shows the distance $D$ at which the radiation can be observed as a function of the Lorentz factor $\gamma$ of the wind for various distances between the neutron star and its companion. For Lorentz factors larger than $\sim 10^{6}$, the radio emission can be observed as far $1 \mathrm{Gpc}$ or more. For a radio emission produced for example by the CMI, an additional amplification factor $\geq 10$ (already in the source frame) is to be taken into account relative to the isotropic power derived above (see Zarka et al. 2004 and references therein). Then, a Lorentz factor of $\sim 10^{5}$ allows detecting $1 \mathrm{Jy}$ emission at $1 \mathrm{Gpc}$ range. Within reasonable ranges for the parameters in Eq. (20), it is possible to produce $1 \mathrm{Jy}$ radio emission at $>1 \mathrm{Gpc}$ range even for $\epsilon=10^{-4}$ and not excessively high $\gamma$ factors.

The power $P_{\text {radio }}$ radiated in the form of radio waves in the companion's wake of about $10^{18} \mathrm{~W}$ for the nominal values of the computation $\left(10^{20-22} \mathrm{~W}\right.$ for a companion at $\left.0.1-0.01 \mathrm{AU}\right)$, is weaker than or comparable to the radio power typically emitted by isolated pulsars $\left(\sim 10^{20}\right.$ to $10^{22} \mathrm{~W}$, as deduced from the ATNF pulsar catalogue, Manchester et al. 2005b,a). But the flux density

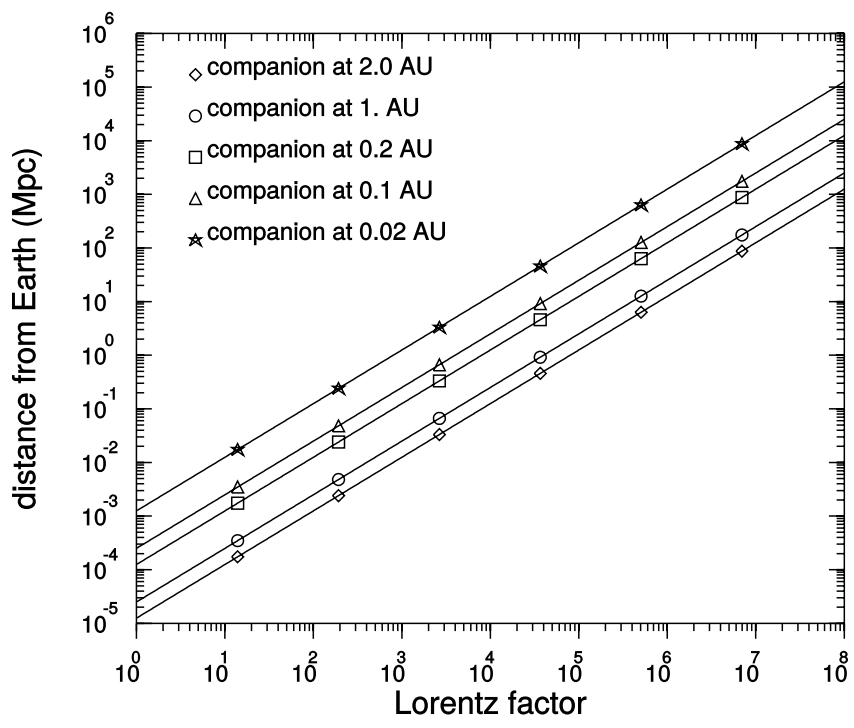

Fig. 8. Distance, expressed in Mpc, at which the radio emission produced in the companion's Alfvén wing can reach a flux density of $1 \mathrm{Jy}$, for various distances from the pulsar to its companion. The values that are not varied are the same as in the text. They correspond to a recycled millisecond pulsar and a coefficient $\epsilon=10^{-3}$. The radio spectrum is assumed to be spread over a spectral range of $1 \mathrm{GHz}$.

is considerably higher for the companion-induced emission because of the very high degree of collimation of the radio beams that is caused by the highly relativistic motion of the sources and leads to an amplification of the flux density by a factor $\sim \gamma^{2}$. This high collimation and not the intrinsically high power of the source permits the beams detection at very large distances.

This phenomenon presents analogies with the high intensity of BL Lac galaxies: they are very bright because we (observers) are aligned with a collimated beam, and because of the relativistic motion of the sources. In the case of BL Lac, the local emission is caused by incoherent synchrotron and inverse Compton radiation at frequencies high above the cyclotron frequency. In the present case, we expect a coherent plasma process (like the CMI) to generate a radiation that, in the frame of the source, is at the local cyclotron frequency. We have not considered the possibility of a high-energy counterpart associated with synchrotron radiation from a hot-plasma component, but this counterpart, emitted by an incoherent process, would be much weaker. (Jupiter's synchrotron emission is five orders of magnitude weaker than its auroral CMI radio emission, Zarka 2004b.)

\section{Possible explanation of remarkable radio transients}

\subsection{Lorimer burst and fast radio bursts}

We propose that the Lorimer burst and FRBs described in the introduction may be the signature of the above-described radio emission from pulsar companions. The inferred and surprisingly large distances deduced in Lorimer (2007) $(0.5 \mathrm{Gpc})$, and even more in Thornton et al. (2013) (1.7-3.2 Gpc), are consistent with the energetics of the pulsar companion signal discussed in the previous section. The corresponding sources would indeed lie in other galaxies, at Gpc distances from us, but as explained above, the exceptionally high collimation of the radio emission allows us to detect these bursts with flux densities of several Jy. This high collimation is also consistent with the rare detection of 
FRBs, although we do not address this point quantitatively here (see rate calculations in Hassall et al. 2013). We discuss here the duration and frequency range of these bursts.

We first consider the duration of FRB signals to be $\sim 5 \mathrm{~ms}$ (part of which may be due to scatter-broadening, Thornton et al. 2013). From Fig. 8, it appears that the Lorentz factor $\gamma_{0}=10^{6}$ would allow for a detection at $\mathrm{Gpc}$ distance. The example displayed in Fig. 5 shows that a companion with an orbital period of a few hours, in a $\gamma_{0}=10^{6}$ wind could provide a signal of adequate duration.

A value of $T_{\text {orb }}$ similar to or higher than a few hours is a reasonable value for the orbital period of a pulsar companion: more than 70 pulsar companions are known with $T_{\text {orb }}<1$ day, $10 \%$ of which have $T_{\text {orb }}<0.1$ day (Manchester et al. 2005b,a), not including possible low-mass planets and asteroids. Moreover, the value of $T_{\text {orb }}$ deduced above is consistent with the fact that no other pulse was observed during the $1.5 \mathrm{~h}$ following the observation of the Lorimer burst and other FRBs.

Considering the asymmetry of the intensity diagram along the circular emision pattern of Fig. 5, the crossing of a pattern corresponding to $\gamma \sim 10^{5}$ is also compatible with the observations, provided that the peak signal from one wing strongly dominates while the other is below the detection threshold.

If the emission is produced by a coherent plasma process such as the CMI, we expect that the radiation is produced along the Alfvén wings at the local cyclotron frequency. Figures 6 and 7 show that we can easily explain an emission at $\sim 1 \mathrm{GHz}$. But the wide spectral range over which FRBs are observed $(\geq 25 \%)$ implies that the emission probably is produced along the Alfvén wings across a wide radial range over which the wind magnetic field and/or $\gamma$ factor vary in this proportion. Another, perhaps more appealing, explanation is that the shift of the velocity distribution of the whole wind along the $v_{x}^{\prime}$ direction in the source frame (i.e. the perpendicular direction, at a particular azimuth) provides an enormous amount of free energy to the CMI, far beyond what is usually available in planetary magnetospheres where a small fraction of the particles are gyrotropically distributed at $v_{\perp} \neq 0$. As a consequence, the emission excited by a pulsar companion could be extremely saturated, generating series of harmonics that possibly result in a quasi-continuous broad spectrum. One of the FRBs reported in Thornton et al. (2013) indeed shows $100 \mathrm{MHz}$-wide bright bands.

\subsection{An event in the Galaxy}

Deneva et al. (2009) reported the discovery of an event composed of three pulsar-like bursts from a source named PSR J1928+15. The bursts occured at intervals of $0.403 \mathrm{~s}$ from each other, with the middle pulse being brighter by an order of magnitude than the other two. The duration of each of the three dedispersed pulses was about $30 \mathrm{~ms}$. The DM is $242 \mathrm{ps} \mathrm{cm}^{-3}$ which, for the galactic coordinates of PSR J1928+15 (longitude $=50.64^{\circ}$, latitude $=-1.03^{\circ}$ ), is compatible with a signal from our Galaxy. In spite of several follow-up observations, the source was not detected again. Deneva et al. (2009) suggested after Cordes \& Shannon (2008) - that it might have been caused by the excitation of a dormant pulsar by accretion of material from an asteroid belt. But they did not provide any physical insight on the underlying mechanism, while Cordes \& Shannon (2008) were mostly concerned with pulsar nullings, instead of isolated pulses from dormant pulsars.

We consider a companion orbiting a pulsar with a $P_{*}=$ $0.403 \mathrm{~s}$ period, in a $\gamma_{0}=10^{3}$ wind at a distance $r=0.01 \mathrm{AU}$.
From Table 1, we can see that (for $a \sim 1000 \mathrm{~km}$ companion), the CMI sources spend $\tau_{-}=80 \mathrm{~ms}$ in the $\delta_{-}$wing, and only $\tau_{+}=0.1 \mathrm{~ms}$ in the other. Therefore, the CMI develops only in the $\delta_{-}$wing. Because $\tau_{-} / \tau_{+}$does not depend on the size $R_{\mathrm{b}}$ of the body, this is true for a smaller object as well. The high-amplitude part of the angular pattern caused by the particle distribution anisotropy of the $\delta_{-}$Alfvén wing can be crossed in about $1.5 \mathrm{~s}$, and this would correspond to 3 pulses of $0.403 \mathrm{~s}$. Each of these pulses would correspond to a slightly different value of $\alpha_{s}$, the second pulse (centre of the emission pattern) being the most intense. Other weaker pulses might have been seen with a lower noise level. The low-amplitude part of the emission angular pattern of the $\delta_{-}$wing would not have been seen either. Of course, the parameters $\gamma_{0}=10^{3}$ and $r=0.01$ AU are simply one possibility among many others. With a single observation, it is not possible to constrain the model more strictly. Nevertheless, the PSR J1928+15 signal is compatible with our pulsar companion model.

PSR J1928+15 is thought to be at a distance $\sim 10 \mathrm{kpc}$, much closer than the sources of the Lorimer burst and FRBs. However, the signal amplitude $\left(S_{\text {peak }} \sim 180 \mathrm{mJy}\right)$ is slightly weaker than that of the very distant FRBs. Therefore, the radio luminosity of this system is much lower. A possible explanation is that its orbiting companion is much smaller than those associated with the extragalactic FRBs. For instance, an asteroid (of radius $\sim \mathrm{km}$ ) would be a possible companion of such a standard pulsar. Because of the smaller size of the companion and according to Eq. (9) the wind plasma would spend less time in the Alfvén wings, allowing less time for the full development of the instability that causes the radio emissions. Because the initial phase of the instability growth is exponential, a variation of this duration by a factor of a few units can imply a difference of several orders of magnitude on the luminosity. The radio signature of such a small companion would not be detectable at extragalactic distances.

\section{Discussion and conclusion}

\subsection{Why we do not necessarily see the radio emission from the central pulsar}

According to our model, the radio emissions from pulsar companions are beamed along the pulsar-companion line. Because the orbital plane of the companion is likely to be very close to the pulsar's equatorial plane, the radio emission from the companion is beamed nearly at a right angle from the rotation axis of the neutron star. Because the radio signals from a pulsar are emitted from its polar cap, a very steep tilt of the magnetic field is needed to be able to see the radio emissions from the pulsar and from the companion. In most cases, we will see only the pulsar signal, even if it possesses a companion, because the emission induced by the companion will never be directed toward Earth. This is why one has detected orbiting planets via a pulsar's radio emission timing without necessarily detecting the planet-induced signal. Conversely, we see only the emission induced by the companion in the case of FRBs because the pulsar's emission is never directed toward Earth (or simply because it is too weak to be detected at extragalactic distances).

Where the magnetic axis (or the companion's orbital plane) is tilted enough relative to the pulsar's rotational axis, however, we may be able to detect both signals, superimposed to each other. Because the companion's signal is very rare (in time) compared with the pulsed signal from the neutron star, it may remain unnoticed except if explicitly searched for, and it will generally 
be invisible in the folded pulsar signal (at the pulsar period) except when it is very strong. Note also that because pulsar timing is based on the variations of distance from the neutron star to the observer, it is mostly efficient for distant companions, thus a low-mass companion orbiting close to the neutron star may well remain undetected by a time-of-arrival analysis.

\subsection{Observational tests}

The theories that have previously been proposed to explain the Lorimer burst and FRBs predict a unique event from a given source, or irregularly repeatable pulses. The latter are not consistent with the large observed DMs, however. In contrast, our explanation based on highly collimated emission from a pulsar companion's wind wake implies that these pulses should be periodically observable at the orbital period of the companion. A decisive test of our theory would thus consist in detecting again a signal with similar characteristics coming from the direction of the source of the Lorimer burst, or of any known FRB, or from PSR J1928+15. We recommend to observe the same regions of the sky again. The difficulty is to observe continuously so as not to miss a pulse with a duration of a few msec that may occur at any time within hours, days, or more.

Another interesting target is the binary pulsar PSR J22220137, which contains a millisecond pulsar orbited by a companion of unknown nature (Deller et al. 2013). As the system is seen nearly edge-on $(\sin i=0.9985 \pm 0.0005)$ and its orbital parameters are known, it is possible to observe it in search for the companion-induced signal around the transit time of the companion between the pulsar and Earth, or even to search for this signal in existing data.

Note that at frequencies about $1 \mathrm{GHz}$, an angular wandering of the source of the order of $10^{-3}$ arcsec and an angular broadening one order of magnitude larger is expected (Cordes 1990). Both effects are much weaker than $1 / \gamma$ and probably do not significantly influence the above discussion. However, intensity fluctuations due to strong scintillation may amplify or attenuate pulses, with an amplitude of fluctuation from one pulse to the other of up to $100 \%$. Thus the detection of the Lorimer burst or of FRBs could have benefited from both a perfect alignment and a constructive scintillation.

Other possible tests of our theory related to the frequency range of the signal discussed in Sect. 4.3 include (i) the existence of periodic (in frequency) intensity fluctuations along the pulse that would be due to emission at harmonics of a given frequency (the fundamental being the frequency interval between bright spots); (ii) the existence of a low-frequency cutoff of the emission below the fundamental frequency predicted in Sect. 4.3; and (iii) the polarization of the radio signal: it is probably strongly circular or elliptical when produced by the CMI (Zarka 2004b).

\subsection{Radio waves from pulsar companions as a probe of pulsar winds}

Up to now, research on very low-mass pulsar companions has led to the discovery of five planets (Wolszczan \& Frail 1992; Thorsett et al. 1993; Bailes et al. 2011) and speculations about orbiting asteroids (Cordes \& Shannon 2008; Deneva et al. 2009; Shannon et al. 2013; Mottez et al. 2013). These objects raise a moderate interest in the field of planetology: they orbit stars that left the main sequence a long time ago (in a state that the Sun will never reach), they are exposed to the neutron star X-rays that probably prevent life from developing, they are embedded in a relativistic wind, and they are very far away from us.
By contrast, the study of these objects is of high interest for the understanding and probing of pulsar winds. If companioninduced radio signals are confirmed, then the underlying theory of Alfvén wings of bodies in a pulsar wind (Mottez \& Heyvaerts 2011a,b; Heyvaerts et al. 2012) would be supported as well. This theory assumes a wind velocity lower than the Alfvén wave velocity (both are very close to $c$ ). This hypothesis, compatible with many theoretical models, is valid only if the pulsar wind at the companion distance is still Poynting-flux dominated. In spite of many discussions (see Bucciantini 2014 for review) there is no convincing proof of this hypothesis. Observational testing of our theory may provide this missing proof and unique measurements of the Lorentz factor of the wind at AU distances, a key ingredient for the theories of dissipation in pulsar winds. The distance range that could be probed is from 10-100 light cylinder radii (otherwise, the companion is destroyed by tidal effects) to a few AU (much closer than the wind termination shock, otherwise at thousands of $\mathrm{AU}$ the companion is practically gravitationally unbound from the neutron star).

If we find that the radio transients that we discussed in Sect. 5 are indeed periodic, the shortest measured period will be the orbital period of the companion $T_{\text {orb }}$. As neutron stars have a narrow range of masses $\left(1-3 M_{\odot}\right)$, we can estimate the companion distance to the star. Then, measured pulse characteristics will allow us to deduce the value of the pulsar wind Lorentz factor $\gamma$ and to know whether the wind is Poynting-flux dominated at the orbital distance of the companion, that is, far away from the light cylinder, and far away from the termination shock.

\section{Appendix A: Orientation of the Alfvén wings relative to the local magnetic field}

The computation of the electric current of Alfvén waves involves a first invariant $\boldsymbol{V}_{s}$, the vector defining the Alfvén characteristics, whose modulus is the speed of an Alfvénic perturbation along these characteristics. With $s= \pm 1$ characterising the two wings, and $\boldsymbol{v}$ and $\boldsymbol{B}$ the perturbed flow velocity and magnetic field

$\boldsymbol{V}_{s}=\boldsymbol{v}-\frac{s \boldsymbol{B}\left(1-\boldsymbol{v}_{0} \cdot \boldsymbol{v} / c^{2}\right)}{\left(\lambda-s \boldsymbol{B}_{0} \cdot \boldsymbol{v} / c^{2}\right)}=\boldsymbol{v}_{0}-\frac{s \boldsymbol{B}_{0}}{\gamma_{0}^{2} \Lambda}$.

The last term is an estimate of the invariant involving only unperturbed wind parameters. The parameter $\lambda$ can be expressed as a function of the unperturbed wind parameters:

$\lambda=\left[\mu_{0} \rho_{0}^{\prime}+c^{-2} B_{0}^{r 2}+c^{-2} \gamma_{0}^{-2} B_{0}^{\phi 2}\right]^{1 / 2}$

where $\rho_{0}^{\prime}$ is the density in the moving frame of the wind (primed quantities refer hereafter to that frame), and

$\Lambda=\lambda-s B_{0}^{r} v_{0} / c^{2} \sim \lambda-s B_{0}^{r} / c$.

The invariant $\boldsymbol{V}_{s}$, related to the theory of uncompressible Alfvén waves, was derived in Mottez \& Heyvaerts (2011b) following a linear approximation for the derivation of the general properties of relativistic Alfvén wings. This invariant was found again in Heyvaerts et al. (2012) in the context of the non-linear theory of relativistic simple Alfvén waves.

Because the direction of the Alfvén wings is those of $\boldsymbol{V}_{s}$ and because $\boldsymbol{v}_{0}=v_{0}^{r}$, the angle $\delta$ between the Alfvén wing and the radial direction is

$\delta_{s}=s \arctan \left(B_{0} / c \Lambda \gamma_{0}^{2}\right)$. 
When $\Lambda$ is expressed as a function of the wind invariants, we find

$\delta_{s}=s \arctan \left[\frac{x}{\gamma_{0}\left(\sqrt{1+x^{2}\left(1+\frac{\gamma_{0}}{\sigma_{0}}\right)}-s\right)}\right]$,

where $\sigma_{0}$ is the magnetization factor defined by

$\sigma_{0}=\frac{\Omega_{*}^{2} \Psi^{2}}{\mu_{0} f c^{3}}$.

The MHD models considering a relativistic radial wind (Arons 2004; Kirk et al. 2009) imply an asymptotic Lorentz factor scaling as

$\sigma_{0} \sim \gamma_{0}^{3}$

and the term $\gamma_{0} / \sigma_{0}$ can be neglected. It is negligible as long as the wind is Poynting-flux dominated, which is the basic assumption that governs this paper. When $\gamma_{0} / \sigma_{0}$ is neglected, we obtain Eq. (4).

Acknowledgements. We thank Michal Bejger (Copernicus Astronomical Center, Polish Academy of Sciences) for suggesting the relevance of pulsar-planet physics to orbiting white dwarfs. This work was partly supported by the CNRS/INSU/PNHE Programme National Hautes Energies.

\section{References}

Arons, J. 2004, Adv. Space Res., 33, 466

Bailes, M., Bates, S. D., Bhalerao, V., et al. 2011, Science, 333, 1717

Bucciantini, N. 2014, Astron. Nachr., 335, 234

Bucciantini, N., Thompson, T. A., Arons, J., Quataert, E., \& Del Zanna, L. 2006, MNRAS, 368, 1717

Budding, E., Slee, O. B., \& Jones, K. 1998, PASA, 15, 183

Cordes, J. M. 1990, in Low Frequency Astrophysics from Space, eds. N. E. Kassim, \& K. W. Weiler (Berlin: Springer Verlag), Lect. Not. Phys., 362, 165

Cordes, J. M., \& Shannon, R. M. 2008, ApJ, 682, 1152

Deller, A. T., Boyles, J., Lorimer, D. R., et al. 2013, ApJ, 770, 145

Deneva, J. S., Cordes, J. M., McLaughlin, M. A., et al. 2009, ApJ, 703, 2259

Farrell, W. M., Lazio, T. J. W., Zarka, P., et al. 2004, Planet. Space Sci., 52, 1469

Freund, H. P., Wong, H. K., Wu, C. S., \& Xu, M. J. 1983, Phys. Fluids, 26, 2263

Goldreich, P., \& Julian, W. H. 1969, ApJ, 157, 869

Hassall, T. E., Keane, E. F., \& Fender, R. P. 2013, MNRAS, 436, 371

Heyvaerts, J., Lehner, T., \& Mottez, F. 2012, A\&A, in press

Huang, Y. F., \& Geng, J. J. 2014, ApJ, 782, L20

Kashiyama, K., Ioka, K., \& Mészáros, P. 2013, ApJ, 776, L39
Keane, E. F., Kramer, M., Lyne, A. G., Stappers, B. W., \& McLaughlin, M. A. 2011, MNRAS, 415, 3065

Keane, E. F., Stappers, B. W., Kramer, M., \& Lyne, A. G. 2012, MNRAS, 425, L71

Kirk, J. G., Lyubarsky, Y., \& Petri, J. 2009, in Astrophys. Space Sci. Lib. 357, ed. W. Becker, 421

Le Queau, D. 1988, in Planetary radio emissions II, Proc. of the Second International Workshop, Graz, Austria, Sept. 7-9, 1987 (A89-39951 17 91), eds. H. O. Rucker, S. J. Bauer, \& B. M. Pedersen (Wien: Verlag der Oesterreichischen Akademie der Wissenschaften), 381

Le Queau, D., \& Roux, A. 1987, Sol. Phys., 111, 59

Loeb, A., Shvartzvald, Y., \& Maoz, D. 2014, MNRAS, in press [arXiv: 1310.2419]

Lorimer, D. 2007, in Chandra Proposal, 2411

Lorimer, D. R., Bailes, M., McLaughlin, M. A., Narkevic, D. J., \& Crawford, F. 2007, Science, 318, 777

Manchester, R. N., Hobbs, G. B., Teoh, A., \& Hobbs, M. 2005a, VizieR Online Data Catalog: VII/245

Manchester, R. N., Hobbs, G. B., Teoh, A., \& Hobbs, M. 2005b, ApJ, 129, 1993

Michel, F. C. 1969, ApJ, 158, 727

Mottez, F., \& Heyvaerts, J. 2011a, A\&A, 532, A22

Mottez, F., \& Heyvaerts, J. 2011b, A\&A, 532, A21

Mottez, F., Bonazzola, S., \& Heyvaerts, J. 2013, A\&A, 555, A126

Neubauer, F. M. 1980, J. Geophys. Res., 85, 1171

Preusse, S., Kopp, A., Büchner, J., \& Motschmann, U. 2006, A\&A, 460, 317

Pritchett, P. L. 1986, Phys. Fluids, 29, 2919

Ransom, S. M., Stairs, I. H., Archibald, A. M., et al. 2014, Nature, 505, 520

Richards, M. T., Waltman, E. B., Ghigo, F. D., \& Richards, D. S. P. 2003, ApJS Ser., 147, 337

Saar, S. H. 1996, in Stellar Surface Structure, eds. K. G. Strassmeier, \& J. L. Linsky, IAU Symp., 176, 237

Saur, J., Neubauer, F. M., Connerney, J. E. P., Zarka, P., \& Kivelson, M. G. 2004, in Plasma interaction of Io with its plasma torus (Cambridge University Press), 537

Savonije, G. J. 1987, Nature, 325, 416

Shannon, R. M., Cordes, J. M., Metcalfe, T. S., et al. 2013, ApJ, 766, 5

Thornton, D., Stappers, B., Bailes, M., et al. 2013, Science, 341, 53

Thorsett, S. E., Arzoumanian, Z., \& Taylor, J. H. 1993, ApJ, 412, L33

Totani, T. 2013, PASJ, 65, L12

van Haarlem, M. P., Wise, M. W., Gunst, A. W., et al. 2013, A\&A, 556, A2

Willes, A. J., \& Wu, K. 2005, A\&A, 432, 1091

Wolszczan, A., \& Frail, D. A. 1992, Nature, 355, 145

Wu, C. S. 1985, Space Sci. Rev., 41, 215

Zarka, P. 2004a, in Extrasolar Planets: Today and Tomorrow, eds. J. Beaulieu, A. Lecavelier Des Etangs, \& C. Terquem, ASP Conf. Ser., 321, 160

Zarka, P. 2004b, Adv. Space Res., 33, 2045

Zarka, P. 2007, Planet. Space Sci., 55, 598

Zarka, P. 2010, in Pathways Towards Habitable Planets, eds. V. Coudé du Foresto, D. M. Gelino, \& I. Ribas, ASP Conf. Ser., 430, 175

Zarka, P., Treumann, R. A., Ryabov, B. P., \& Ryabov, V. B. 2001, Ap\&SS, 277, 293

Zarka, P., Cecconi, B., \& Kurth, W. S. 2004, J. Geophys. Res., 109, 9

Zhang, B. 2014, ApJ, 780, L21 